\title{
Philosophiques
}

\section{Procédés d'inversion chez Poulain de la Barre : pour un concept d'efféminage}

\section{Marie-Frédérique Pellegrin}

Volume 44, numéro 2, automne 2017

Les nouveaux horizons du féminisme dans la philosophie francophone

URI : https://id.erudit.org/iderudit/1042330ar

DOI : https://doi.org/10.7202/1042330ar

Aller au sommaire du numéro

\section{Éditeur(s)}

Société de philosophie du Québec

ISSN

0316-2923 (imprimé)

1492-1391 (numérique)

Découvrir la revue

Citer cet article

Pellegrin, M.-F. (2017). Procédés d'inversion chez Poulain de la Barre : pour un concept d'efféminage. Philosophiques, 44(2), 193-208.

https://doi.org/10.7202/1042330ar
Résumé de l'article

Poulain de la Barre fait un usage original des procédés d'inversion (utopies et autres mondes à l'envers) tels qu'on les trouve en littérature et en philosophie, afin de démontrer l'égalité des sexes. Chez lui, l'inversion des valeurs ne vise pas seulement à remettre en cause le bien-fondé de la domination masculine et à louer une supériorité féminine en matière de moeurs et d'intelligence. Cette inversion entend abolir toute hiérarchisation entre les sexes. Tout d'abord l'auteur émancipe le genre du sexe (il y a des hommes mous et des femmes viriles, telles les amazones). Ensuite il prône un efféminage de tous les êtres humains. Le concept d'efféminage que nous forgeons est à distinguer de celui d'efféminement, qui implique l'idée d'une féminisation au sens d'une inclination vers des valeurs considérées comme féminines d'un point de vue culturel. L'efféminage est une prescription morale et sociale à devenir femme au sens d'un retour à l'état prélapsaire parfait dont les femmes sont plus héritières que les hommes car elles ont moins été touchées par le péché originel. L'efféminage ne consiste donc pas à devenir femme au sens d'une simple féminisation - sociale - des moeurs. L'efféminage fait signe vers l'égalité et non vers une supériorité féminine que sous-entend l'impératif d'efféminement. Le concept d'efféminage permet donc de penser, de manière explicite, le genre contre le sexe, non pas comme inversion mais comme construction ou plutôt reconstruction d'une autre nature humaine.
Tous droits réservés @ Société de philosophie du Québec, 2017
Ce document est protégé par la loi sur le droit d'auteur. L’utilisation des services d'Érudit (y compris la reproduction) est assujettie à sa politique d'utilisation que vous pouvez consulter en ligne.

https://apropos.erudit.org/fr/usagers/politique-dutilisation/ 


\title{
Procédés d'inversion chez Poulain de la Barre: pour un concept d'efféminage
}

\author{
MARIE-FRÉDÉRIQUE PELLEGRIN \\ Faculté de philosophie \\ Université Jean Moulin-Lyon III \\ UMR 5317
}

RÉSUMÉ. - Poulain de la Barre fait un usage original des procédés d'inversion (utopies et autres mondes à l'envers) tels qu'on les trouve en littérature et en philosophie, afin de démontrer l'égalité des sexes. Chez lui, l'inversion des valeurs ne vise pas seulement à remettre en cause le bien-fondé de la domination masculine et à louer une supériorité féminine en matière de mœurs et d'intelligence. Cette inversion entend abolir toute hiérarchisation entre les sexes. Tout d'abord l'auteur émancipe le genre du sexe (il y a des hommes mous et des femmes viriles, telles les amazones). Ensuite il prône un efféminage de tous les êtres humains. Le concept d'efféminage que nous forgeons est à distinguer de celui d'efféminement, qui implique l'idée d'une féminisation au sens d'une inclination vers des valeurs considérées comme féminines d'un point de vue culturel. L'efféminage est une prescription morale et sociale à devenir femme au sens d'un retour à l'état prélapsaire parfait dont les femmes sont plus héritières que les hommes car elles ont moins été touchées par le péché originel. L'efféminage ne consiste donc pas à devenir femme au sens d'une simple féminisation — sociale — des mœurs. L'efféminage fait signe vers l'égalité et non vers une supériorité féminine que sous-entend l'impératif d'efféminement. Le concept d'efféminage permet donc de penser, de manière explicite, le genre contre le sexe, non pas comme inversion mais comme construction ou plutôt reconstruction d'une autre nature humaine.

ABSTRACT. - Poulain de la Barre made original use of methods of inversion (utopias and other upside-down worlds) such as are found in literature and in philosophy, in order to demonstrate the equality of the sexes. In his work, the inversion of values is aimed not only at calling into question the legitimacy of male dominance and at praising feminine superiority in matters of morals and intelligence. It is an attempt to abolish any organization of the sexes into a hierarchy. In the first place, the author liberates gender from sex (there are delicate men and virile women, such as the Amazons). Next, he advocates the "efféminage" of all human beings. The concept of "efféminage" that we are constructing here is to be distinguished from that of "efféminement", which implies the idea of feminization in the sense of an inclination toward values considered feminine from a cultural perspective. "Efféminage" is a moral and social prescription to become a woman in the sense of a return to the perfect prelapsarian state of which women are more properly the inheritors because they have been less affected by original sin. "Efféminage" does not then consist in becoming a woman in the sense of a simple social feminization of morals. "Efféminage" gestures toward equality and not toward the feminine superiority implied by the imperative of "efféminement". The concept of "efféminage" allows us then to think explicitly about gender in distinction with sex, not as an inversion but as a construction or rather a reconstruction of another human nature. 
En démontrant philosophiquement l'égalité entre les sexes, Poulain de la Barre (I647-I723) rompt avec les arguments philogynes traditionnels. Dans leur immense majorité en effet, ceux-ci défendent une supériorité des femmes sur les hommes. Le schème égalitaire est peu présent dans cette littérature au Moyen Âge et sous l'Ancien Régime, et il est quasiment absent dans des textes plus nettement philosophiques ${ }^{1}$. Dans un tel schéma, comparer les hommes et les femmes aboutit nécessairement à créer une hiérarchie ${ }^{2}$. Les arguments traditionnels mobilisés par cette littérature paraissent donc inopérants pour penser l'égalité. Même si l'influence de la philosophie cartésienne permet à Poulain de renouveler profondément ses démonstrations en faveur des femmes, on trouve pourtant certains motifs venus de cette topique philogyne médiévale et moderne. Leur étude permet de constater que l'auteur les mobilise autrement, en vue de défendre une évolution profonde des modèles anthropologiques dominants.

Cet usage renouvelé de procédés argumentatifs anciens s'explique d'abord par le travail critique opéré sur eux et sur soi par l'auteur. Il constate en effet que des femmes "tout ce qu'en ont dit les hommes doit être suspect» car ils sont «juges et parties" ${ }^{3}$. La question de l'objectivité de son discours se pose à tout homme qui entend défendre les femmes, pour des raisons qui tiennent à la spécificité du préjugé misogyne: il existe en tout temps et en tout lieu, il structure l'ensemble de nos représentations et jugements ${ }^{4}$. Cela s'explique, selon Poulain, par le fait que ce préjugé-là est fondateur de l'humanité postlapsaire. Il naît avec la faute d'Adam. Il est même la marque de cette faute: le premier geste de l'Adam déchu étant de dominer sa compagne ${ }^{5}$. On peut toujours faire l'éloge des femmes, comme le fait la

1. La seule exception réelle à ce constat est le court traité de Marie de Gournay: L'égalité des hommes et des femmes, datant de 1622.

2. Les titres mêmes des ouvrages relevant de cette littérature philogyne font signe vers une lecture hiérarchique des rapports entre sexes. Voici le relevé indicatif de M. Angenot: «Le triomphe des dames", "Le triomphe du sexe ", "Le champion des femmes ", "Apothéose du sexe ", "Apologie du beau sexe ", "De la supériorité des femmes ", "Défense des femmes ». Les champions des femmes, Montréal, Presses universitaires du Québec, I977, p. 4.

3. F. Poulain de la Barre, De l'égalité des sexes, p. 93 in De l'égalité des deux sexes; De l'éducation des dames; De l'excellence des hommes, éd. critique de M.-F. Pellegrin, Paris, Vrin, 20 I I (toutes les références à Poulain se font dans cette édition).

4. À la manière cartésienne, Poulain souligne que d'une opinion reçue de tou.te.s, la raison doit d'autant plus se méfier: «[D]u moment que je prétends que l'opinion commune est un préjugé et une erreur, tous ceux qui y sont engagés deviennent mes parties, et par conséquent récusables, n'y ayant plus que la raison qui nous puisse juger. Et de dire qu'un sentiment reçu de tous les hommes ne peut être faux, c'est répondre ce qui est en question. Le peu de gens qu'il y a qui suivent la raison, et la peine que l'on a pour la découvrir, nous apprennent assez à nous défier de ce qui est le plus universellement reçu et pratiqué, comme étant peut-être l'effet le plus naturel de la corruption des hommes, et des passions qui les gouvernent» (De l'excellence des hommes, p. 379).

5. "C'est ce que Dieu voulut faire entendre à Ève lorsqu'il lui dit qu'elle allait être sous la puissance de son mari, l'avertissant par ces paroles que le péché auquel elle avait eu part, le 
littérature philogyne depuis des siècles. Mais c'est encore pour tout "champion des femmes ${ }^{6}{ }^{»}$ se situer dans une position de surplomb qui ne peut convenir à celui qui entend affirmer une égalité absolue entre les deux sexes. Il faut donc trouver les moyens intellectuels et rhétoriques de rompre avec un discours philogyne qui porte la marque de la masculinité de son auteur. Ce décentrement, nécessaire par rapport à ce que l'on est, passe notamment par un usage philosophique original de procédés topiques. Parmi ces derniers, le plus efficace pour opérer un tel décentrement est celui de l'inversion. On en trouve de nombreuses illustrations dans la littérature philogyne. Poulain lui-même y a recours de différentes manières, dont la plus remarquable est sa proposition d'inverser tous les propos sur les femmes et sur les hommes dans la Bible ${ }^{7}$, afin de restituer au texte sacré sa valeur universelle, quel que soit son sexe. Poulain fait ainsi tout autre chose de ces procédés classiques, et l'inversion devient le principe même d'un renversement total des valeurs.

\section{La tradition du monde à l'envers: le royaume des amazones}

Le plus significatif de ces procédés d'inversion se trouve dans la fiction dite de «monde à l'envers». Il s'agit d'un récit littéraire et philosophique qui décrit un monde où les valeurs et les positions sociales ou politiques sont inversées par rapport au monde existant. Cette fiction implique un renversement des valeurs qui fait que ce qui est en haut se retrouve en bas, et réciproquement. Elle rappelle certaines pratiques sociales comme le carnaval. Depuis le Moyen Âge, ce dernier constitue un moment festif où l'ordre social et sexuel s'abolit, créant un désordre (ou un nouvel ordre) temporaire qui opère par inversion entre dominants et dominés. Elle est proche également du genre de l'utopie, dont elle est l'ancêtre et auquel elle sert souvent de moyen ${ }^{8}$.

dérèglerait tellement que, sans se soucier de l'égalité qui était entre eux, il prendrait sujet d'exercer sur elle un empire de domination. C'est là en effet le seul sens raisonnable et digne de l'Écriture que l'on puisse donner à ce passage, que nous avons supposé pour vrai, vous serez sous la puissance de votre mari, etc., car ne pouvant signifier ni une loi positive, ni une punition formelle, comme nous l'avons montré, il faut que ce soit la prédiction d'un malheur, qui peut néanmoins passer pour une peine imposée " (De l'excellence des hommes, p. 3 I 4 ). "C'est pourquoi ayant reçu du premier l'exemple de dominer sur les femmes, il n'est pas si mal aisé de comprendre qu'ils [les hommes] l'aient porté et conservé partout où ils se sont répandus» (ibidem, p. 379).

6. L'expression, utilisée au Moyen Âge, donne son titre à l'ouvrage de M. Angenot qui examine quatre-vingts traités affirmant la supériorité des femmes sur les hommes de I 400 à I 800 (op. cit.).

7. Voir notre article, «Une lecture philogyne des grands textes est-elle possible? François Poulain de la Barre (I647-I723) ", Les arts de lire des philosophes modernes, Presses de l'Université Laval, 20I 5.

8. Pour l'ensemble des critères qui distinguent et rapprochent les deux genres du monde imaginaire et du monde utopique, voir P.-F. Moreau, Le récit utopique. Droit naturel et fonctionnement de l'État, Paris, Presses universitaires de France, I 982. 
Les fictions littéraires ou philosophiques recourent souvent à ce type de retournement où l'ordre communément admis, les hiérarchies qui le structurent et les valeurs qui y sont associées sont effacés afin d'interroger leur légitimité et leur sens9. La pièce d'Aristophane L'Assemblée des femmes constitue un exemple de ce procédé d'inversion ${ }^{10}$. Réunies sur l'agora, les femmes décident des mesures à prendre pour sauver la cité sans en référer aux hommes qui se réveillent le lendemain, privés de leur pouvoir de décision et mis devant le fait accompli. La pièce se termine par une scène de banquet où les femmes fêtent ce nouvel ordre social et politique qu'elles ont instauré. Comme la pièce vise à dénoncer la politique athénienne de l'époque, cette cité où les femmes mènent les affaires publiques va mal. Le règne des femmes est néfaste car il inverse un état naturel des choses. Le procédé est ici utilisé de manière critique. Dans d'autres récits au contraire, le renversement des valeurs y est présenté comme positif. Puisque la hiérarchie du monde existant est en faveur des hommes, l'utopie peut ainsi proposer une inversion de cette hiérarchie c'est-à-dire une fiction où les femmes l'emportent sur les hommes, soit par leurs qualités intrinsèques, soit par le pouvoir (intellectuel, social ou politique) qui leur est attribué. La Cité des dames de Christine de Pisan (I 405) ouvrage souvent présenté comme inaugural du genre littéraire de la défense des femmes, décrit par exemple une cité parfaite construite par des "dames», allégories des vertus humaines ${ }^{11}$. L'auteure le présente comme un "royaume de fémynie ${ }^{12}$ » où les qualités intrinsèques des bâtisseuses s'expriment dans la perfection de l'œuvre accomplie. La gynocratie constitue ici une alternative heureuse.

Qu'elle soit décrite de manière positive ou de manière négative, la fiction de monde à l'envers suppose toujours que l'inférieur et le supérieur, le dominant et le dominé intervertissent leurs places. Elle maintient donc un principe hiérarchique. De ce fait, elle ne semble pas très bien adaptée à une démonstration égalitaire, et l'on ne voit pas, au premier abord, comment Poulain pourrait la faire servir à son dessein démonstratif. Jamais d'ailleurs, il ne prône un gouvernement de femmes, de quelque ordre qu'il soit, dans

9. Pour en donner quelques exemples, nous citons P.-F. Moreau: «Ce thème de l'inversion des usages est présent dès le récit de Raphaël Hythlodée, où les utilisations qui sont faites de l'or et des diamants abolissent le respect qui leur est porté en Europe. Dans le royaume d'Antangil, le roi est une personne dénuée de pouvoir. Cyrano évoque un royaume où les enfants commandent et où les adultes obéissent. Les habitants de la terre australe craignent si peu la mort qu'ils se suicident en masse. Chez Restif, le procédé structure un chapitre entier: les Mégapatagons qui habitent aux antipodes, ont des coiffures qui ressemblent à nos souliers, et leurs chaussures ont la forme d'un chapeau» (op. cit., p. 6I).

10. Aristophane, "L’Assemblée des femmes" dans Théâtre complet II, Paris, Gallimard, 2002 [1965].

11. Christine de Pisan, La Cité des dames, Paris, Stock, I986.

12.Voir K. Wilson-Chevalier, Royaume de fémynie. Pouvoirs, contraintes, espaces de liberté des femmes, de la Renaissance à la Fronde, Paris, Champion, I999, p. I I. 
ses trois traités féministes. Il revendique bien toujours un partage de l'autorité entre les deux sexes.

Il mobilise pourtant ce procédé au travers d'un type de monde à l'envers spécifique, celui du royaume des amazones. Ce thème a été incorporé aux argumentaires philogynes au XIV ${ }^{\mathrm{e}}$ siècle et se développe ensuite, notamment à la Renaissance ${ }^{13}$. Décrivant une société régie par les femmes, ce mythe opère un décentrement dont la visée est la possibilité d'un regard critique sur les sociétés phallocratiques ${ }^{14}$. Dans l'occurrence la plus anecdotique de ce mythe des amazones chez Poulain, on voit bien comment l'inversion permet de prendre du recul par rapport aux réflexes misogynes. Poulain l'utilise ici pour interroger les différences supposées de comportement, c'està-dire de valeurs morales et sociales, selon qu'on est homme ou femme.

Caton ce sage et sévère Critique priait souvent les Dieux de lui pardonner, s'il avait été assez imprudent pour confier le moindre secret à une femme. Le bon homme avait à cœur un fait fameux de l'Histoire Romaine, dont les Antiquaires se servent comme d'un grand argument pour montrer le peu de retenue des femmes. Un Enfant de douze ans pressé par sa mère de lui dire la résolution du Sénat, où il avait assisté, inventa pour sa défaite, qu'on avait arrêté de donner plusieurs femmes à chaque mari. Elle l'alla dire aussitôt à ses voisines, pour prendre des mesures avec elles; et toute la Ville le sut au bout d'une demiheure. Je voudrais bien savoir, ce que ferait un pauvre mari, si dans un État où les femmes seraient les Maîtresses, comme dans celui des Amazones, on lui venait rapporter, qu'il aurait été résolu au Conseil, de donner à chaque homme un compagnon: sans doute qu'il n'en dirait mot. ${ }^{15}$.

Lorsqu'un bouleversement dans les coutumes et les habitudes sociales survient, tous les êtres humains réagissent de la même manière. Le commérage, défaut traditionnellement attribué aux femmes, comme l'étymologie même du terme l'indique, se transformerait en "compérage » si les hommes étaient surpris par un changement d'organisation de la communauté aussi important que l'introduction de la polyandrie. Le royaume des amazones sert ici de toile de fond permettant l'inversion des points de vue. Il figure une organisation sociale où les femmes font les lois et où les hommes sont soumis à leurs décisions. Il montre donc au passage à quel point il est redoutable d'être soumis aux lois que l'on n'a pas faites. Mais le cœur de l'argument est la disproportion entre la nouvelle étonnante (l'instauration de la polygamie) et le reproche fait aux femmes de s'en émouvoir et de colporter la nouvelle.

13. Voir G. Leduc et S. Steinberg, Réalités et représentations des amazones, Paris, L'Harmattan, 2008.

14. Dans La Cité des dames, de Christine de Pisan, déjà évoquée, l'édification de la cité parfaite commence par un travail de terrassement. L'auteure explique qu'ayant déblayé les pierres noires symbolisant certains écrits misogynes comme celui de Jean de Meung, cette cité est établie sur de nouvelles fondations, dont certaines pierres reprennent les noms des amazones les plus connues: Thamiris, Menalippe, Hippolyte et Penthésilée.

15. De l'égalité des deux sexes, p. I44. 
Ici les amazones constituent une métonymie de toute forme de gynocratie, ce qui explique que cette référence soit mobilisée à plusieurs reprises.

Ce thème amazonien permet en effet de réfléchir à un gouvernement des femmes dans la sphère publique. Il subvertit les attributs essentiels du masculin en présentant des femmes autonomes puisqu'elles guerroient, qu'elles ne se marient pas et qu'elles dirigent politiquement. Le mythe des amazones exemplifie bien un schéma d'inversion entre dominants et dominés :

Tous les raisonnements, de ceux qui soutiennent que le beau Sexe n'est pas si noble, ni si excellent que le nôtre, sont fondés sur ce que les hommes étant les maîtres, on croit que tout est pour eux; et je suis assuré qu'on croirait tout le contraire, encore plus fortement, c'est-à-dire, que les hommes ne sont que pour les femmes, si elles avaient toute l'autorité, comme dans l'Empire des Amazones ${ }^{16}$.

Dans un tel empire, les femmes sont donc les maîtresses des hommes et elles exemplifient un pouvoir féminin et matriarcal. Ce motif des amazones permet bien d'opérer un décentrement de type relativiste. Il aboutit à une mise à distance des valeurs sociales par le soi. On réalise alors qu'on donne toujours sens et ordre au monde à partir du point de vue masculin. Mais ce motif dit également qu'on donne sens et ordre au monde à partir du point de vue du dominant. L'exemple des amazones permet donc de mettre en doute le caractère naturel du commandement masculin, il n'abolit cependant pas une structure sociale fondée sur l'opposition entre dominant et dominé. Il peut mettre en cause une hiérarchie mais pas l'idée de hiérarchie, puisqu'il la maintient en l'inversant. Ce procédé est donc capable d'ébranler le préjugé misogyne, mais il ne semble pas pouvoir produire l'idée d'égalité à lui seul.

Que les hommes soient soumis aux femmes ou qu'ils soient exclus de ce royaume amazonien, toute l'autorité va ici aux femmes. Les deux versions du thème existent en effet dans la littérature philogyne: ou bien il s'agit d'un royaume mixte avec des hommes soumis aux femmes; ou bien il s'agit d'un royaume entièrement féminin (les rencontres avec l'autre sexe n'ayant que la reproduction pour finalité et les enfants mâles résultant de ces unions étant exclus du royaume). La description donnée par Poulain d'un lieu où "les hommes ne sont que pour les femmes " pourrait convenir à ces deux versions du mythe. Comme le procédé vise à inverser les rôles, on peut cependant avancer qu'il envisage ici une société mixte où les hommes sont soumis aux femmes. Il y a bien inversion des rôles pour les deux sexes cohabitant dans une même communauté socio-politique. Ce point est important, car l'exclusion absolue des hommes empêcherait toute réflexion sur la mixité, réflexion au cœur de la pensée de Poulain. 
L'inversion des rôles sur le plan social et politique permet d'interroger le caractère, naturel ou non, de la phallocratie. Il pose donc parallèlement la question de la possibilité et de l'efficience d'une gynocratie. Il est ici intéressant de comparer l'usage de ce mythe des amazones chez Poulain et chez Spinoza ${ }^{17}$, qui sont contemporains et réfléchissent tous deux à des changements sociaux et politiques de grande ampleur. Les prémisses sont les mêmes: à l'exception des amazones, tous les gouvernements humains semblent avoir été masculins. Mais le procédé d'inversion n'est pas là pour remettre en cause ce constat chez Spinoza. Dans la description qu'il en propose en effet, le royaume des amazones est un règne de la violence qui va contre toute paix civile:

Partout sur Terre où l'on trouve des hommes et des femmes, nous voyons les hommes régner, les femmes être gouvernées, et les deux sexes vivre dans la concorde selon ce principe. Et inversement, les Amazones qui, à ce que l'on rapporte, régnèrent autrefois, ne toléraient pas que des hommes demeurent sur leur territoire, n'élevaient que des filles, et tuaient les mâles qu'elles avaient enfantés.

L'inversion brutale introduit l'intolérance et le meurtre, qui sont ici les principes mêmes du gouvernement. Il est significatif que Spinoza présente une vision non mixte de cette gynocratie. Tout ce paragraphe sur les femmes (l'unique de tout son corpus d'ailleurs!) est structuré autour de l'alternative entre gouvernement des hommes et gouvernement des femmes. L'éventualité d'un gouvernement mixte, le seul qui intéresse Poulain, est immédiatement écartée: "que des hommes et des femmes règnent ensemble, cela ne s'est produit nulle part». Ce constat d'expérience est confirmé ensuite par une affirmation définitive: "il n'est pas possible que les deux sexes gouvernent à parité». Chez Spinoza, l'expérience écrase toute mise à distance critique. Il n'y a jamais eu de gouvernement mixte ou de gynocratie, ce qui prouve qu'il n'est pas naturel que les femmes gouvernent. La seule exception à ce constat suppose que ce principe naturel soit violé (par la mise à mort des mâles).

Lorsque Spinoza étend sa réflexion en sortant de l'exemple des amazones, et émet l'hypothèse d'une entité politique mixte mais gouvernée par les femmes, il précise que, pour qu'un tel gouvernement existe, il faudrait que les hommes reçoivent "une éducation propre à limiter leur ingéniosité ». Il faudrait donc contrer, de manière artificielle, une qualité naturelle aux hommes pour que ce pouvoir féminin dans une société mixte soit tenable. Les femmes sont inférieures politiquement aux hommes, un gouvernement égalitaire mixte nécessiterait de rabaisser ces derniers au niveau d'ingéniosité des femmes.

17. Spinoza, Traité politique, XI, 4, Paris, Presses universitaires de France, 2005 , p. 273. (Toutes les références à Spinoza proviennent de cette page sauf mention contraire. Nous ne faisons donc pas d'appels de note pour les références spinoziennes quand elles viennent de ce paragraphe.) 
Ce paragraphe sur la place des femmes dans la cité se situe à l'orée d'une réflexion sur la démocratie (que le lecteur ne pourra jamais découvrir puisque l'ouvrage est inachevé). Or, au paragraphe précédant celui-ci, Spinoza précise qu'il ne va pas traiter de tous les genres de démocratie mais d'un seul, qui exclut les étrangers, les femmes, les serviteurs, les enfants, les pupilles et "ceux qui sont taxés d'infamie pour un forfait ou un genre de vie honteux ${ }^{18}$. Ces différentes catégories ne sont évidemment pas à mettre sur le même plan, mais elles ont bien en partage, pour des raisons diverses, l'inaptitude politique. Si les femmes sont soumises aux hommes dans n'importe quel type d'État, et même dans un régime qui serait fondé sur l'égalité entre citoyens, "cette situation provient de leur faiblesse». Leur inaptitude est donc naturelle. Et Spinoza de conclure qu'il est "permis d'affirmer sans réserve que les femmes n'ont pas par nature un droit égal à celui des hommes, qu'elles leur cèdent nécessairement le pas, et que par conséquent il n'est pas possible que les deux sexes gouvernent à parité ». Cette conclusion, péremptoire, clôt la possibilité même d'un débat.

Ce contre-point spinozien rappelle plusieurs éléments importants: tout d'abord, il illustre la vivacité du thème du monde à l'envers, expérience de pensée qui décentre du réel en permettant d'envisager d'autres organisations socio-politiques. Il est donc utile à toute réflexion qui cherche de nouvelles formes de vivre-ensemble. Il montre ensuite que l'idée d'égalité est limitée dans l'analyse spinozienne. Le procédé d'inversion ne parvient pas ici à produire cette véritable expérience de pensée qui sortirait des modèles existants. Alors même qu'il s'agit d'une fiction, d'une utopie permettant de penser autrement ce qui est et de penser autrement que ce qui est, elle se trouve en effet défaite par le réel. C'est "l'expérience même", le fait que nulle part "parmi tant de nations si diverses » les femmes n'ont part au gouvernement qui invalide l'idée d'un gouvernement mixte ou d'une gynocratie. La manière dont Spinoza use de ce procédé d'inversion en annihile la fécondité. Elle ne l'aide pas à penser autre chose que ce qui s'est toujours fait. Elle ne remplit pas non plus pleinement sa fonction d'utopie puisqu'elle peut être contredite par le réel.

Il est à cet égard intéressant de mettre son analyse en parallèle avec celle du personnage misogyne mis en scène par Poulain dans son troisième traité intitulé De l'excellence des hommes. Cet ouvrage fait parler un contempteur fictif des femmes, partisan de la supériorité des hommes par rapport aux femmes. Deux voix philogynes lui répondent ${ }^{19}$, mais c'est son discours même qui est intéressant, car il est très proche de l'argumentaire spinozien.

18. Spinoza, op. cit., p. 27I.

19. Voir notre introduction à cet ouvrage, p. 29I-293 in De l'égalité des deux sexes; De l'éducation des dames; De l'excellence des hommes, op. cit. 
L'on a vu des nations disputer ensemble de la noblesse; mais l'on n'a jamais ouï dire que les femmes aient prétendu d'être aussi nobles que les hommes. On dit qu'il y a eu autrefois une troupe de femmes qui s'avisèrent de faire bande à part, et de former un état qui subsista quelque temps: mais l'histoire ne nous apprend point que ce fut par un sentiment d'égalité, mais seulement pour se délivrer de la servitude où le Mariage les engageait. Or il est visible que si l'opinion de l'égalité des sexes était une erreur de prévention, les femmes du moins la reconnaîtraient, et en même temps qu'elles se plaignent de la dureté dont les hommes usent à leur égard, elles les accuseraient d'être injustes par une ignorance grossière ils eussent rendu leur condition plus avantageuse et plus douce. Puisque si elles n'ont point de part aux sciences ni aux emplois avec les hommes, c'est que ceux-ci sont persuadés que cela leur appartient comme un apanage de leur sexe. Et les femmes sont elles-mêmes si fortement convaincues de leur inégalité et de leur incapacité, qu'elles se font une vertu non seulement de supporter la dépendance où elles sont, mais encore de croire qu'elle est fondée sur la différence que la nature a mise entre elles et les hommes $^{20}$.

La discussion d'un gouvernement des femmes n'a jamais été réelle (contrairement à celle portant sur la capacité politique exclusive ou non de la noblesse). Ce sujet n'est pas un enjeu politique concret. La meilleure preuve en est que les femmes mêmes ne se sont jamais révoltées contre le sort qui leur était fait. Elles n’ont jamais remis en cause la soumission à l'égard des hommes que l'on constate partout et de tout temps. S'éloignant alors de ce constat qui pourrait être spinozien, le texte avance un argument fondamental dans la réflexion sur l'égalité des sexes. Alors même qu'un misogyne est mis en scène, c'est en effet une thèse essentielle de Poulain en faveur de l'égalité qui est mobilisée. Les amazones en effet, selon ce personnage, se sont éloignées des hommes pour ne plus subir le joug du mariage. Longuement ailleurs, Poulain explique à quel point les contrats de mariage ne sont pas de véritables contrats entre parties majeures et libres de contracter, mais des pactes de soumission pour l'épouse à l'égard de son mari ${ }^{21}$. Si les femmes ne dénoncent pas leur place subordonnée dans la société, cette place correspond bien à une inégalité naturelle.

20. De l'excellence des hommes, p. 340-34I.

21. «Pour ce qui est de la société du mariage, elle n'est composée que de deux personnes, dont l'une par conséquent ne peut user de commandement et de contrainte à l'égard de l'autre. Cette société n'est point établie sur la crainte, mais sur l'amour. [...] Lorsqu'ils conviennent de vivre ensemble, c'est de pure volonté et dans un âge où ils peuvent avoir autant de raison et d'expérience l'un que l'autre. Quand les femmes en auraient moins, le Contrat qu'elles font étant très libre, les hommes n'ont de pouvoir qu'autant qu'elles leur en veulent céder. Je mets toujours à part la coutume. Ainsi l'autorité, le commandement et la puissance sur le corps et sur les biens est aussi grande dans la femme que dans l'homme. [...] De sorte que l'on peut fort bien conclure que les femmes ne dépendent des hommes que par les lois qu'ils ont faites pour leur avantage particulier ", De l'excellence des hommes, p. 3 I3-3 I 4. 
Ainsi, même lorsqu'il met en scène un misogyne, Poulain trouve à l'exemple des amazones une valeur démonstrative pour la défense des femmes. Les amazones constituent bien un cas de révolte contre la domination masculine que l'immense majorité des femmes subit à son époque: le mariage et la soumission à autrui qu'il engendre.

Lorsque l'exemple des amazones est une seconde fois mobilisé dans ses démonstrations explicitement philogynes dans De l'égalité des deux sexes, il appuie non plus un argument d'inversion des hiérarchies, mais une thèse égalitaire. Par là, il fait sortir cette référence des discours sur la supériorité (des hommes ou des femmes) et l'acclimate à sa perspective philosophique égalitaire:

Ce qui peut faire penser que si l'on exerçait également les deux Sexes, l'un acquerrait peut-être autant de vigueur que l'autre; ce que l'on a vu autrefois dans une République, où la Lutte et les exercices leurs étaient communs: on rapporte le même des Amazones qui sont au Midi de l'Amérique ${ }^{22}$.

L'exemple est mobilisé pour réfuter l'idée d'une plus grande faiblesse physique des femmes. L'inversion se produit en décrivant un peuple de femmes guerrières. Mais l'association de cet exemple à une allusion à la belle cité platonicienne et/ou à Sparte fait qu'il ne s'agit plus simplement d'inverser une hiérarchie. Les femmes ont montré leur force dans un royaume de guerrières, et certaines pratiques éducatives égalitaires confirment que les deux sexes ont les mêmes capacités corporelles. La plus grande faiblesse physique des femmes est culturelle et acquise. Elle est liée à la manière masculine de percevoir leur corps et au type d'éducation qu'en conséquence on leur prodigue.

Il serait intéressant de savoir si l'allusion à une république où autrefois les exercices physiques étaient les mêmes pour les deux sexes fait référence à Platon ou à Sparte. Dans la seconde hypothèse, en effet, il s'agirait d'un cas historique réel d'une éducation égalitaire en ce qui concerne le développement des forces corporelles de tou.te.s. Il ferait sortir clairement de l'utopie. Mais la république platonicienne peut aussi jouer ce rôle. Elle s'inspire en effet fortement du régime spartiate, donc de l'existant, pour proposer une vision non genrée des corps qui n'est pas totalement utopique ${ }^{23}$. Cette seconde occurrence du monde à l'envers amazonien adossée à un second exemple historique (puisqu'à l'époque de Poulain, on considère que ce royaume féminin a réellement existé) montre que l'auteur abandonne l'utopie. Le royaume des amazones est un monde à l'envers, mais il n'est pas

22. De l'égalité des deux sexes, p. I28. Nous soulignons en italique.

23. Et par ailleurs, comme le souligne P.-F. Moreau, la cité platonicienne ne correspond pas tout à fait au genre de l'utopie car "elle n'est pas un récit et elle ne décrit pas le tableau d'une société fonctionnant actuellement (même si c'est imaginairement)» (op. cit., p. 26). 
fictif. Il est considéré comme ayant existé24 . L'égalité entre les êtres humains est un principe qui a déjà fonctionné. Exception comme chez Spinoza, le mode d'organisation sociale des amazones met à mal l'idée d'une supériorité naturelle des hommes sur les femmes.

\section{Création d'un nouveau monde à l'envers: le royaume efféminé}

Mais l'amazone est un exemple de femme forte. Il reste d'une certaine manière dans le registre des catalogues de femmes hors du commun qui emplissent les écrits philogynes de forme traditionnelle. Une telle figure est exceptionnelle, elle n'est qu'une exception. Dans la dernière occurrence étudiée, l'exemple a permis de penser un type important d'égalité, l'égalité de force corporelle entre les deux sexes. Mais à partir de lui il faut produire une inversion générale, qui amène à un relativisme plus poussé. Le but est un véritable renversement des valeurs, ce que montre la suite du raisonnement de Poulain juste après ce passage sur les amazones:

C'est donc assez afin qu'ils donnent naissance à un troisième, qu'il y ait quelques organes dans l'un qui ne soient pas dans l'autre. Il n'est pas besoin pour cela, comme on se le figure, que les femmes aient moins de force et de vigueur que les hommes. Et comme il n'y a que l'expérience qui puisse bien faire juger de cette distinction, ne trouve-t-on pas que les femmes sont mêlées comme nous; il y en a de fortes et de faibles dans les deux parties: les hommes élevés dans la mollesse sont souvent pires que les femmes, et ploient d'abord sous le travail: mais quand ils y sont endurcis par nécessité ou autrement, ils deviennent égaux, et quelquefois supérieurs aux autres ${ }^{25}$.

La force associée aux hommes et la faiblesse associée aux femmes sont des données réversibles. Il y a des hommes mous (la mollesse étant en principe la manifestation psycho-morale des humeurs féminines dans la médecine inspirée d'Hippocrate), tout comme il existe des femmes viriles — comme on vient de le voir. L'égalité et le déploiement de certaines qualités individuelles (car il est bien entendu que tout le monde n'a pas exactement les mêmes capacités en toutes choses ${ }^{26}$ ) se construisent, notamment par l'éducation. Les êtres humains sont "mêlés »: ce mélange qui fait qu'on ne peut distinguer assurément la supériorité d'un sexe sur l'autre, appelle à la mixité.

Que les femmes soient traditionnellement décrites comme faibles explique que pour louer les femmes exceptionnelles on les appelle «femmes

24. Voir G. Leduc, op. cit., p. I4-I 8 ; plusieurs récits de voyage du XVI ${ }^{e}$ siècle font état de royaumes féminins: le mythe parvient à façonner la réalité. Sur l'existence historique et/ou mythique des amazones, voir A. Mayor, Les amazones, Paris, La Découverte, 2017.

25. Ibidem.

26. "Quoi qu'il en soit, afin que deux personnes soient égales dans une société, il n'est pas nécessaire qu'elles puissent faire la même chose, ou qu'elles la fassent de la même manière. C'est assez qu'elles en puissent faire d'équivalentes. » De l'excellence des hommes, p. 379. 
fortes $»^{27} »-$ et ce depuis la Bible, qui en met quelques-unes en exergue. Cela signifie que, lorsqu'on veut louer une femme, on lui attribue une qualité masculine. Et c'est ce réflexe-là qu'il faut abolir selon Poulain, comme nous allons le voir. Il participe d'une misogynie plus subtile puisqu'il prend place dans un éloge de la femme, mais en lui assignant des valeurs traditionnellement associées aux hommes.

Si le thème de la femme forte n'est pas un argument suffisant pour une défense des femmes, c'est donc parce que d'une part elle constitue une exception, et parce que d'autre part elle est louée à l'aune de valeurs masculines. Une analyse critique de ces valeurs s'impose. Cette enquête minutieuse exhume trois éléments fondamentaux. Tout d'abord, ces valeurs sont misogynes; ensuite elles ne permettent pas d'établir des jugements fondés; enfin elles doivent être inversées, cette inversion n'ayant pas pour but une nouvelle hiérarchie (contraire à la première) mais un retour à l'équilibre de valeurs qui ne dépendent pas du sexe.

Le premier point vient d'être démontré au travers d'une réflexion critique sur la louange de la femme forte. Le deuxième est explicité au moyen d'une réflexion sur le corps. Comme les corps, masculin et féminin, ne se distinguent pas réellement ${ }^{28}$, ils ne sont pas porteurs de valeurs différentes. S'il y a des femmes fortes et des hommes faibles, cela signifie bien que genrer les corps constitue une erreur quand cette démarche s'appuie sur la différence sexuelle. Le fort et le faible, le viril et le mou, le brutal et le délicat ne sont pas des caractéristiques marquant une frontière entre les sexes. Le mélange de ces qualités est intersexuel. Le troisième point va porter plus loin l'analyse du deuxième pour y proposer un remède. Les valeurs associées au masculin sont des coutumes qui ne reposent pas sur des qualités masculines essentielles, naturelles. Elles s'expliquent par leur éducation. Ces valeurs ne sont donc pas masculines mais associées aux hommes par une longue coutume valorisant toutes les formes de force et donc de domination. Leur corps et leur esprit ont été modelés par rapport à ces valeurs supposément viriles. Mais, encore une fois, un homme élevé dans la mollesse est mou. Il n'est pas fort par essence.

Dès lors, il convient d'interroger la hiérarchie de ces valeurs:

L'on ne doit donc faire aucun fond sur certaines expressions ordinaires tirées de l'état présent des deux Sexes. Lorsqu'on veut blâmer un homme avec moquerie, comme ayant peu de courage, de résolution et de fermeté, on l'appelle efféminé, comme si on voulait dire, qu'il est aussi lâche, et aussi mou

27. Voir par exemple La gallerie des femmes fortes, du père Le Moyne (2 vol., Paris, Compagnie des Marchands Libraires du Palais, I665).

28. Il ne faut pas "porter trop loin la différence des Sexes. On la doit restreindre dans le dessein que Dieu a eu de former les hommes par le concours de deux personnes, et n'en admettre qu'autant qu'il est nécessaire pour cet effet". De l'égalité des deux sexes, p. I28. La différence entre hommes et femmes se réduit donc aux seuls organes sexuels. 
qu'une femme. Au contraire, pour louer une femme qui n'est pas du commun à cause de son courage, de sa force, ou de son esprit, on dit, que c'est un homme. Ces expressions si avantageuses aux hommes ne contribuent pas peu à entretenir la haute idée qu'on a d'eux; faute de savoir qu'elles ne sont que vraisemblables; et que leur vérité suppose indifféremment la nature, ou la coutume, et qu'ainsi elles sont purement contingentes et arbitraires. La vertu, la douceur et l'honnêteté étant si particulières aux femmes, si leur sexe n'avait pas été si peu considéré, lorsqu'on aurait voulu signifier avec éloge qu'un homme a ces qualités en un degré éminent, on aurait dit, c'est une femme, s'il avait plu aux hommes d'établir cet usage dans le discours ${ }^{29}$.

L'inversion des valeurs est une opération d'efféminage. Ce néologisme me paraît le plus à même de décrire la révolution axiologique proposée par Poulain. Le qualificatif d'efféminé a toujours été péjoratif. Cela signifie qu'attribuer une dimension féminine à un comportement d'homme, c'est le dénigrer. Afin de remettre en cause des appréciations morales fondées sur une valorisation des qualités masculines (acquises), il faut que les qualités féminines (acquises) comme le doux ou le délicat reçoivent une appréciation positive. Le monde à l'envers ici proposé est un monde où l'efféminage est loué. Non plus une société de femmes fortes (comme dans le royaume des amazones) mais une société d'efféminé(e)s, que l'on soit homme ou femme.

Ce procédé d'inversion revêt un sens qui le rattache à une pensée du genre, même si le terme est évidemment anachronique à l'époque de Poulain:

Confondez l'injustice et l'aveuglement des hommes; et faites que par un agréable retour ce terme d'efféminé, qui a été un terme d'injure, devienne un terme d'honneur; et qu'au lieu que jusqu'ici on a cru faire grâce et un éloge aux femmes de leur dire qu'elles ont un esprit mâle, on loue les hommes au contraire, en disant qu'ils ont l'esprit d'une femme ${ }^{30}$.

Les qualités dites féminines ou masculines sont indépendantes du sexe de la personne qui les possède. Elles constituent des positions genrées sans lien direct avec les organes sexuels. L'efféminage des hommes est possible puisque leurs qualités viriles sont purement coutumières. Or cet efféminage est nécessaire pour s'acheminer vers une société égalitaire. Car c'est le point le plus crucial: un monde efféminé ne serait pas un monde où les femmes gouvernent les hommes, ce serait un monde qui aurait renoncé à systématiquement privilégier les pseudo-valeurs associées au masculin et à dénigrer tout ce qui est associé au féminin.

\section{De l'inversion à l'absence de hiérarchisation}

C'est donc le principe même de hiérarchie et l'action de hiérarchiser qu'il faut combattre. L'exemple des amazones a permis de réfuter une partie du

29. Ibidem, p. I29.

30. De l'éducation des dames, p. I7I. 
préjugé misogyne, celui qui porte sur le caractère profondément sexué des corps. En aidant à penser une force féminine, il fait signe vers son envers, la faiblesse masculine (c'est-à-dire dans les catégories traditionnelles, son caractère efféminé). La démonstration peut alors établir que cette faiblesselà est une vertu et non un vice en la ramenant à l'état prélaspaire de l'être humain. Il permet donc de glisser du physiologique au moral, l'un ne déterminant plus l'autre; l'un n'indiquant nulle valeur universelle à l'autre. Mais il est évidemment important de souligner que l'efféminage est un principe intellectuel, et pas seulement moral. Comme l'indique le texte, il s'agit avant tout d'efféminer l'esprit, de pouvoir dire d'un homme avec louange qu'il a l'esprit d'une femme. Ce n'est que si cesse la différence entre des manières masculines et des manières féminines de penser que l'égalité peut être restaurée. Cette différence provient de la coutume qui altère le principe premier selon lequel «l'esprit n'a point de sexe ${ }^{31}{ }^{1}$.

Ainsi est-il possible que les hommes puissent s'efféminer sans bafouer leur nature, puisque les valeurs dites viriles sont purement construites. De plus, et c'est l'essentiel, l'efféminage est un principe purgatif. Il vise à sortir de la phallocratie, mais le but n'est pas d'aller vers une gynocratie. Le procédé d'inversion qui paraissait net (valorisation des valeurs féminines contre les valeurs masculines) n'en est pas un. L'efféminage vise en effet un retour à l'équilibre dans la détermination des valeurs. Il apure l'idée d'une supériorité des valeurs masculines, mais il ne rend pas les hommes féminins, il les rend à eux-mêmes, c'est-à-dire à une nature humaine parfaite, commune aux deux sexes. Il n'est pas un nouveau principe hiérarchique fondé sur la différence sexuelle.

En effet, devenir plus féminin, c'est revenir à la nature humaine d'avant le péché. On a dit que le signe de la faute d'Adam est qu'il se met à dominer Ève: il introduit une hiérarchie fondée sur la différence sexuelle. Dans l'analyse de Poulain en effet, tout préjugé se définit comme un sentiment de supériorité indu ${ }^{32}$. Le premier sentiment de ce type a été celui d'Adam dans sa relation nouvelle avec Ève après le péché. Ainsi, l'origine de tous les préjugés est le préjugé misogyne. On peut donc en réalité appeler le préjugé misogyne un préjugé hiérarchique dont découlent d'autres préjugés qui sont autant de rapports de domination: des lettrés sur les ignorants et des nobles sur le commun. Tout préjugé est un principe de hiérarchisation. Mais la nature prime de l'être humain était étrangère à ce désir d'asservissement. Le fait de s'efféminer marque donc une table rase à l'égard du préjugé misogyne, un

31. De l'égalité des deux sexes, p. 99.

32. C'est ce que Poulain appelle la supériorité de volonté par opposition à la supériorité d'esprit. Il n'y a que Dieu qui puisse imposer la première aux hommes, car ils doivent lui être soumis sans condition. La seconde au contraire, fondée sur des rapports égalitaires et rationnels, est librement consentie quand on reconnaît que quelqu'un a plus de mérite que soi. Dans ce cas, "ce n'est pas à celui qui commande que nous obéissons, c'est à notre propre raison qui est avertie de son devoir». De l'excellence des hommes, p. 309. 
retour à une nature première commune à tous les êtres humains. S'efféminer, c'est revenir aux qualités naturelles de l'humanité, c'est-à-dire à ses caractéristiques premières, prélapsaires, qui sont toujours propices à la concorde et au développement de la raison.

Si ce retour passe par un processus d'efféminage, c'est donc parce que ce sont d'abord les hommes qui ont été tyranniques envers leurs égales que sont les femmes. On pourrait penser que les choses ont changé puisque le préjugé misogyne est désormais quasiment universel, y compris chez les femmes, constate Poulain ${ }^{33}$. Mais en fait, il faut aussi que les hommes s'efféminent, car les femmes sont malgré tout moins touchées par le péché originel que les hommes selon l'auteur ${ }^{34}$. En l'état actuel de la civilisation, les femmes sont plus proches que les hommes de l'état prime de l'être humain. Si elles sont moins imbues que les hommes de ce que l'on peut désormais appeler le préjugé hiérarchique, c'est d'abord parce qu'elles ne sont pas éduquées (donc elles sont plus épargnées par le préjugé misogyne partout présent dans l'instruction des enfants). C'est ensuite parce qu'elles ont conservé des valeurs morales authentiques et de bon sens (on doit rechercher la paix, l'amour et la conservation de soi et des autres, contre les valeurs guerrières masculines). Ces deux explications ne renvoient donc pas à une nature féminine spécifique, mais à la nature originelle des êtres humains. Pour ces deux raisons, en définitive, les femmes exemplifient souvent mieux la nature humaine que les hommes. S'efféminer est un retour à la vertu première, celle qui pense des rapports humains sans désir de domination. En se construisant un nouveau genre, les hommes ne renient pas leur sexe, ils redécouvrent une humanité authentique c'est-à-dire des valeurs pacifiques et civilisatrices.

L'idée qu'il faut s'efféminer - dont je tire le concept d' "efféminage " — telle que Poulain permet de la penser s'avère donc très différente du procédé d'inversion traditionnel qui demande aux hommes de se convertir à des valeurs féminines. S'il s'appuie sur ce topos du caractère pacificateur et civilisateur des femmes, ce topos semblant à cet égard relever des procédés d'inversion, il a en réalité une signification autre. Il constitue un nouveau

33. «Les femmes sont elles mêmes si fortement convaincues de leur inégalité et de leur incapacité, qu'elles se font une vertu non seulement de supporter la dépendance où elles sont, mais encore de croire qu'elle est fondée sur la différence que la nature a mise entre elles et les hommes. Je me souviens encore fort bien que lorsque le livre de l'égalité commença à paraître, il n'y eut que les Précieuses qui le reçurent avec applaudissement disant qu'on leur faisait quelque justice; d'autres le firent valoir seulement parce qu'il flattait leur vanité: mais tout le reste en parla comme d'un paradoxe qui avait plus de galanterie que de vérité, n'osant pas le condamner tout à fait, parce qu'il leur était favorable. »Ibidem, p. 34I.

34. Ce point est évoqué au travers de la discussion (topique dans la littérature philogyne et misogyne) de la responsabilité respective d'Adam et Ève dans le péché originel. Poulain tranche: "On ne peut pas nier qu'Ève ne fut moins coupable qu'Adam.» Ibid., p. 307. Si les femmes sont devenues dépendantes des hommes [...] il faut conclure qu'elles ne le sont point par les règles générales de la nature, puisqu'elles ne le deviennent que par accident. ", Iibid., p. 306. 
programme, une pédagogie originale pour l'émancipation des femmes. Ici, l'inversion est un retour qui nie des différences essentielles entre hommes et femmes fondées sur la distinction des sexes sur lesquelles l'étude de l'homme s'est appuyée jusque là. Ce concept d'efféminage est donc à distinguer de celui d'efféminement, qui implique l'idée d'une féminisation au sens d'une inclination vers des valeurs considérées comme féminines d'un point de vue culturel. L'efféminage est une prescription morale et sociale à devenir femme au sens d'un retour à l'état prélapsaire, et non à devenir femme au sens d'une simple féminisation - sociale — des mœurs. L'efféminage fait signe vers l'égalité, et non vers une supériorité féminine que sous-entend l'impératif d'efféminement.

Le concept d'efféminage permet donc de penser, de manière explicite, le genre contre le sexe, non pas comme inversion, mais comme construction ou plutôt reconstruction d'une autre nature humaine. L'efféminage n'est donc pas ce qui est propre aux femmes ni même précisément le féminin, mais le naturellement humain. Ce concept fonde donc une nouvelle anthropologie. 